\title{
SUSPENSION OF \\ DIAMINODIPHENYLSULPHONE IN LEPROSY
}

\author{
A. T. Roy.
}

When the proprietary sulphones proved successful in the treatment of leprosy, attention naturally turned to the use of the parent sulphone, $4: 4^{\prime}$ diaminodiphenylsulphone (DDS or DADPS) as a possibly more economic method of therapy.

Cochrane (I) started injecting a suspension of DDS intradermally into the macules. Later on, on skin estimation, he found that DDS could be recovered from the skin tissues in which the sulphone was deposited. This finding made him abandon intradermal injections and adopt the subcutaneous injection. He found very favourable results from subcutaneous injections of $25 \%$ suspension of DDS in groundnut oil. This report helped all leprologists to get rid of the alarmingly heavy cost of the proprietary preparations. Molesworth and Narayanswami (2), advised by Cochrane, took up the experiment. They used a $20 \%$ suspension in place of $25 \%$ in purified and deodorized neutral cocoanut oil and reported favourable results in 100 lepromatous cases, after a year's trial.

In August, I950, Muir selected I70 cases for DDS injection. Out of these, I 40 cases, 75 males, 39 females and 26 children, have been included in this report. All of them were lepromatous cases. The plea for writing this is to show the progress of these cases after the completion of I year's treatment.

\section{Preparation of the Suspension.}

A $20 \%$ suspension was made first in hydnocarpus oil and sterilized in an autoclave at $15 \mathrm{lb}$. pressure for half an hour. A cork with two glass tubes, one straight and long reaching the bottom, and the other short and bent, were fitted to the container of the sterilized suspension. This bottle was often shaken and emptied in a small sterilized glass bowl, in small quantities at a time. From this bowl the syringes were filled by a laboratory assistant who, after drawing in and out the suspension several times, to make the injectable quantities of the same uniformity, handed them over to the injector. Next the hydnocarpus oil was changed to refined cocoanut oil with the same percentage of DDS.

Dosage ANd Method of InJeCtion.

All injections were given bi-weekly and subcutaneously in the 
extensor surfaces of arms and thighs in rotation. All the patients, male, female and children, got the same dose of I cc $(.2 \mathrm{grm}$.$) of$ the suspension. Observation of reaction, dosage, examination for depot formations and detailed bacteriological examinations were done only by the doctor in charge of the experiment, to ensure accuracy and uniformity of the result.

\section{Method of Bacteriological Examination AND Assessment of Progress.}

This was done before the experiment was started, and repeated after 12 months. Five smears were examined from the most infected positive sites of each case on both occasions, and the average result of the five smears from +4 to $+I$ was taken as the bacteriological index, e.g. the most positive smear was counted as +4 and the least positive was counted as $+\mathrm{I},+2$ and +3 for less or more midstages. By adding the counts of the 5 smears, and dividing these 5, the bacteriological index was assessed.

"Partly negative" = Those cases who have improved much, but revealed only a few bacilli in all the 5 smears, e.g. (4 in 30 fields).

"Much improved" = This was reckoned when the present bacteriological index was less by I or more, e.g. if the initial B.I. was +4 and became less than +3 .

"Moderately improved" was given to those just less by $+\mathrm{I}$ from the initial bacteriological index, e.g. +4 to +3 , or +3 to +2 .

"Slightly improved" = Those whose bacteriological index had improved by less than $\mathrm{I}$.

"Stationary " and "Worse" are self explanatory.

\section{RESULTS.}

(I) Cases males Io, 30, 54, 72 and I03, females 22, 25, 30, 36 and 55 (total $7.1 \%$ ), have become partly negative after an average of $\mathrm{I} 8.64$ and $\mathrm{I} 8.2 \mathrm{gm}$. of DDS respectively. The maximum improvement is in case F.36. The Bacteriological Index came down from +4 to $+4 / 5^{\text {ths, }}$ and in case Male I03, B.I. from $\mathrm{I}+$ to 2 bacilli in all the 5 smears.

(2) 37 cases $(26.7 \%)$ improved a great deal. The Bacteriological Index became less by more than I. Cases Male 8, I8, 49, $74,78,97$, and female 4,9 have improved more than the other cases of this group. The Bacteriological Index became less than I only.

(3) I 8 cases ( $\mathrm{I} 2.8 \%$ ) became moderately improved. The B.I. lessened after I year's treatment just by one. 
(4) 47 cases $(33.5 \%)$ improved slightly.

(.5) $20(\mathrm{I} 4.3 \%)$ and I6, or II.4\%, remained stationary or became worse respectively.

TABLE 1.

Results and average total dose of DDS in males and females separately.

\begin{tabular}{|c|c|c|c|c|c|c|}
\hline \multirow{2}{*}{ Advancement. } & \multicolumn{2}{|c|}{ No. } & \multirow{2}{*}{$\begin{array}{c}\text { Total } \\
\%\end{array}$} & \multicolumn{2}{|c|}{ Dosage Avg. in Grm. } & \multirow{2}{*}{ Period } \\
\hline & Male & Female & & Male & Female & \\
\hline Partly negative ... & 7 & 5 & 8.5 & г 8.64 & 18.2 & I 2 months \\
\hline Much improved ... & 17 & I0 & 19.3 & 18.6 & 19.2 & , \\
\hline Mod. improved ... & Io & 8 & 12.8 & 19.1 & 19.7 & .. \\
\hline Slightly Improved & 30 & 17 & $33 \cdot 5$ & I 8.9 & I8. I & .. \\
\hline Stationary $\quad \ldots$ & It & 4 & $14 \cdot 3$ & I9. I & 19.7 & .. \\
\hline Worse $\quad \ldots$ & $\frac{9}{80}$ & $\frac{7}{51}$ & 11.4 & 19.8 & 19.7 & .. \\
\hline
\end{tabular}

TABI.E 2--see pages 76,77 and 78 .

\section{Observation and Discussion.}

\section{SUSPENSION OF DDS POWDER.}

Cochrane used groundnut oil, though he has not given any details regarding its purification, acidity etc. It may well be surmised that he used purified and injectable oil. Molesworth et al changed the groundnut oil to the acid free purified cocoanut oil, which was thinner than groundnut oil and could be injected easily, using a medium sized (Gauze 23) hypodermic needle. The author used filtered groundnut oil, hydnocarpus oil and acid free, purified and deodorised cocoanut oil, all sterilised. Groundnut oil was found to be the thickest, and difficulties were encountered specially for mass treatment. Hydnocarpus and cocoanut oil could be used easily.

\section{Depots.}

The formation of depots was found in all. Groundnut oil took a longer time to be absorbed, but if massaged well, as has been done here, the number of depots become minimised. It is interesting to note that cases M. 44, 45, 85 and II4 always formed depots after DDS injection, be the suspension in cocoanut, hydnocarpus or groundnut oil.

Doses:-As has been mentioned, I cc of $20 \%$ ( $0.2 \mathrm{grm})$ was all along given twice a week (per week $.4 \mathrm{grm}$ ), irrespective of age 
TABLE 2.

Showing the results of the examinations in details, sex by sex.

PARTLY NEGATIVE

Males

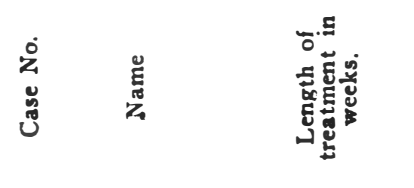

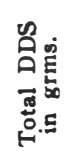

$47 \frac{1}{2}$

$45 \frac{1}{2}$

Opindra

Nobin

Amrit

Jogu

$\cdots$

... 50

8 Khudu

Bokul

Mongol

Bhim

Durjon

Chuttu

Sorbo

Pundee

Horinath

Akhoy

Monu

Nokul

Nimai

Gobardhan

Bihai

99 Motilal

... 32

... 20

... $\quad 51 \frac{1}{2}$

… 45

... 38

$\begin{array}{ll}\ldots & 49 \\ \ldots & 48\end{array}$

50

$47 \frac{1}{2}$

49

$47 \frac{1}{2}$

49
$50 \frac{1}{2}$

$51 \frac{1}{2}$

50

4 Gopal

33 Akhey

35 Konthiram ... $\begin{array}{ll}48 & 19.2 \\ 50 \frac{1}{2} & 20.2\end{array}$

$50 \frac{1}{2}$
$50 \frac{1}{2}$

20.2
20.2
15.6

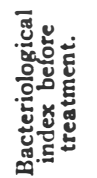

$+2$

18.2

20.4

18.8

20.2

20.4

I 2.8

8.0
20.6

20.2

18.2

19.2

19.6

19.2

20.4

I 9.4

19.6

19.4
19.4

19.6

19.6

20.6

20.4

$+31 / 5$
$+32 / 5$

$+32 / 5$
$+33 / 5$

$31 / 5$
$24 / 5$

$+\mathrm{I}$

$+23 / 5$

$+13 / 5$
$+11 / 5$

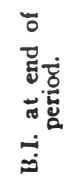

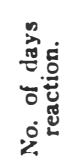

$\dot{3}$
$\dot{z}$
0
$u$
$u$

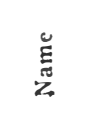

$4 / 5 \quad-$

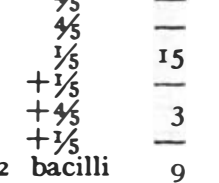

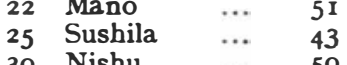

30 Nishu ... 50

36 Kehsori $\ldots . \quad 32 \frac{1}{2}$

55 Jotsna

2 bacilli 9

\section{MUCH IMPROVED}

$\begin{array}{llr}+31 / 5 & +\mathrm{I} & 24 \\ +34 / 5 & +\mathrm{I} & 3 \mathrm{I} \\ +4 & +24 / 5 & - \\ +3 & +\mathrm{I} & - \\ +32 / 5 & +\mathrm{I} 1 / 5 & \mathrm{I} 8 \\ +34 / 5 & +21 / 5 & \mathrm{I} 5 \\ +4 & +\mathrm{I} 1 / 5 & 7 \\ +31 / 5 & +\mathrm{I} 4 / 5 & 2 \mathrm{I} \\ +4 & +21 / 5 & - \\ +22 / 5 & +\mathrm{I} 1 / 5 & 2 \mathrm{I} \\ +2 & +\mathrm{I} & 9 \\ +3 \mathrm{I} / 5 & +\mathrm{I} & - \\ +21 / 5 & +\mathrm{I} & - \\ +4 & +23 / 5 & - \\ +4 & +\mathrm{I} 4 / 5 & - \\ +21 / 5 & +\mathrm{I} & - \\ +4 & +\mathrm{I} / 5 & -\end{array}$

MODERATELY IMPROVED

$\begin{array}{rr}21 / 5 & 14 \\ 22 / 5 & 3 \\ 23 / 5 & 3\end{array}$

$\begin{array}{ll}26 & \text { Indi } \\ 28 & \text { Rupi }\end{array}$

$\begin{array}{ll}28 & \text { Rupi } \\ 35 & \text { Mongli }\end{array}$

50
$49 \frac{1}{2}$
50
PARTLY NEGATIVE

Females
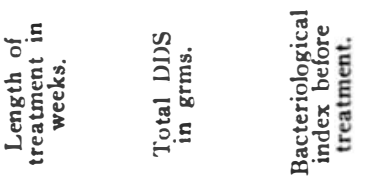

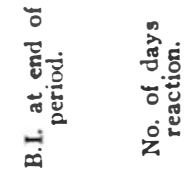

$+21 / 5$
$+31 / 5$
+2
+4
$+22 / 5$

$\begin{array}{ll}4 / 5 & - \\ 4 / 5 & 52 \\ 3 / 5 & - \\ 4 / 5 & 61 \\ 4 / 5 & -\end{array}$

$\begin{array}{rllll}4 & \text { Subni } & \ldots & 46 & 18.4 \\ 9 & \text { Singho } & \ldots & 48 & 19.2 \\ \text { I0 } & \text { Ambika } & \ldots & 47 & 18.8 \\ \text { I } 7 & \text { Jhabri } & \ldots & 49 \frac{1}{2} & 19.8 \\ 24 & \text { Thelia } & \ldots & 45 \frac{1}{2} & 18.1 \\ 31 & \text { Kemola } & \ldots & 49 & 19.6 \\ 34 & \text { Ruou } & \ldots & 50 & 20.4 \\ 38 & \text { Hira } & \ldots & 47 & 18.8 \\ 42 & \text { Sauri } & \ldots & 46 & 18.4 \\ 50 & \text { Jamila } & \ldots & 51 \frac{1}{2} & 20.6\end{array}$

+245
$+22 / 5$
$+33 / 5$
$+31 / 5$
+345
+4
+4
+4
$+31 / 5$
$23 / 5$

$\begin{array}{rr}+1 & 31 \\ +1 & 7 \\ +13 / 5 & 10 \\ +13 / 5 & - \\ +2 & 21 \\ +24 / 5 & 17 \\ +23 / 5 & 10 \\ +24 / 5 & 21 \\ 115 / 5 & 49 \\ 11 / 5 & -\end{array}$

20.4
19.8

$+12 / 5$
$+33 / 5$

$+2 / 5 \quad 7$

$\begin{array}{llll}20.4 & +33 / 5 & +22 / 5 & \text { 10 } \\ 24 / 5 & +24 / 5 & 3\end{array}$ 


$\begin{array}{rll}45 & \text { Thakurlal } & \ldots \\ 63 & \text { Noru } & \ldots \\ 89 & \text { Chamru } & \ldots \\ 95 & \text { Biswaneth } & \ldots \\ 98 & \text { Purna } & \ldots \\ \text { IOO } & \text { Jogeswar } & \ldots \\ \text { IOI } & \text { Bank } & \ldots\end{array}$

$\begin{array}{llll}\text { r8.7 } & +2 & +\mathrm{r} & 24 \\ 18.6 & +2 & +\mathrm{r} & \mathrm{I} 8 \\ \mathrm{r} 5.5 & +4 & +3 & 5 \mathrm{I} \\ 20.6 & +\mathrm{r} 3 / 5 & +3 / 5 & - \\ 18.2 & +32 / 5 & +\mathrm{I} 2 / 5 & 38 \\ 20.2 & +\mathrm{r} 2 / 5 & +2 / 5 & - \\ 19.6 & +33 / 5 & +23 / 5 & -\end{array}$

$\begin{array}{llll}39 & \text { Nilmoni } & \ldots & 42 \\ 43 & \text { Babi } & \ldots & 5 \text { I } \\ 49 & \text { Taramoni } & \ldots & 50 \\ 5 \text { I } & \text { Habi } & \ldots & 49 \\ 52 & \text { Khetu } & \ldots & 5 \text { I } \frac{1}{2}\end{array}$

16.8
20.4
20.2
19.8
20.6

$\begin{array}{lllll}\text { I } & \text { Radha } & \ldots & 49 & \text { I9.6 } \\ 6 & \text { Charu } & \ldots & 48 & \text { I9.2 }\end{array}$

9 Rakhal $\quad \cdots \quad 48 \quad 19.2$

9 Rakhal

Gulba $\quad \cdots \quad 47 \frac{1}{2}$

I Baneswar ...

20 Damri

27 Bhuru

28 Bijoy

32 Sarjuram ...

7 Bhaskar ...

48 Kashinath ...

5o Nilamber ...

52 Lalmohan ...

5 Ghasiram ...

60 Menon

$\begin{array}{rr}+34 / 5 & \text { I4 } \\ +22 / 5 & 7 \\ +3 & 3 \\ +\mathrm{r} & \mathrm{I} 4 \\ +31 / 5 & - \\ +31 / 5 & \mathrm{r} 4 \\ +33 / 5 & 7 \\ +24 / 5 & 2 \mathrm{I} \\ +34 / 5 & 24 \\ +32 / 5 & \mathrm{I} 4 \\ +32 / 5 & 45 \\ +31 / 5 & 3 \mathrm{I} \\ +4 / 5 & 35 \\ 335 & 2 \mathrm{I} \\ 455 & - \\ +415 & 2 \mathrm{I} \\ +22 / 5 & -\end{array}$

\section{SLIGHTLY IMPROVED}

Males

$\begin{array}{rlll}67 & \text { Horish } & \ldots & 5 \mathrm{I} \\ 68 & \text { Dhonu } & \ldots & 50 \\ 77 & \text { Kitu } & \ldots & 50 \frac{1}{2} \\ 80 & \text { Purna } & \ldots & 5 \mathrm{I} \\ 82 & \text { Rothu } & \ldots & 49 \frac{1}{2} \\ 86 & \text { Ebadot } & \ldots & 48 \\ 90 & \text { Dibaker } & \ldots & 46 \\ 93 & \text { Suru } & \ldots & 48 \frac{1}{2} \\ 96 & \text { Ronjit } & \ldots & 5 \mathrm{I} \\ \text { I02 } & \text { Kartik } & \ldots & 5 \mathrm{I} \frac{1}{2} \\ \text { I07 } & \text { Sibsankar } & \ldots & 48 \frac{1}{2} \\ \text { I08 } & \text { Kopildee } & \ldots & 49 \frac{1}{2} \\ \text { I14 } & \text { Lachmi } & \ldots & 5 \mathrm{I}\end{array}$

\section{4}

20.4

20.2

20.4
19.8

19.2

$\mathrm{I} 8.4$

19.4

20.4

20.6

$\div 9.3$

19.8

20.4

\section{SI.IGHTLY IMPROVED}

$\begin{aligned} \text { I } & \text { Golapi } \\ 6 & \text { Alomoni } \\ 7 & \text { Sundora } \\ \text { 5 } & \text { Opi } \\ \text { I } 2 & \text { Phudi } \\ \text { I4 } & \text { Buchi } \\ \text { I5 } & \text { Rojoni } \\ \text { I8 } & \text { Rudun } \\ \text { 19 } & \text { Rongi } \\ 23 & \text { Phulu } \\ 27 & \text { Binoti } \\ 29 & \text { Champa } \\ 37 & \text { Khandhi } \\ 46 & \text { Dugi } \\ 47 & \text { Gendu } \\ 48 & \text { Adu } \\ 53 & \text { Balika }\end{aligned}$

$\begin{array}{ll}\ldots & 50 \frac{1}{2} \\ \ldots & 48 \frac{1}{2} \\ \ldots & 46 \\ \ldots & 48 \\ \ldots & 42 \\ \ldots & 51 \frac{1}{2} \\ \ldots & 46 \\ \ldots & 47 \\ \ldots & 51 \\ \ldots & 51 \\ \ldots & 49 \\ \ldots & 47 \frac{1}{2} \\ \ldots & 50 \\ \ldots & 46 \\ \ldots & 51 \\ \ldots & 49 \frac{1}{2} \\ \ldots & 37 \frac{1}{2}\end{array}$

20.6
19.3
18.4
19.2
16.8
20.6
18.4
18.8
20.4
20.4
19.6
19.4
20.4
18.4
20.4
19.8
15.4

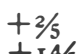

$+14 / 5$ $+14 / 5$

$+14 / 5$

$+2 / 5$

$+1 / 5$
+4

$+34 / 5$
+

$+1 / 5$
$+34 / 5$

$+34 / 5$

+4
$+21 / 5$

$+21 / 5$
$+11 / 5$

$+31 / 5$

$+24 / 5$

$+22 / 5$

$+4$

$\begin{array}{cr}2 \text { granules } & 3 \\ +\mathrm{I} & \mathrm{IO} \\ +\mathrm{I} 2 / 5 & \mathrm{I} 7 \\ +\mathrm{I} 2 / 5 & \mathrm{I} 7 \\ +\mathrm{I} / 5 & 2 \mathrm{I} \\ +1 / 5 & - \\ +33 / 5 & 52 \\ +33 / 5 & \mathrm{I} 4 \\ +1 / 5 & - \\ +32 / 5 & - \\ 31 / 5 & \mathrm{I4} \\ 13 / 5 & 38 \\ 4 / 5 & - \\ 24 / 5 & 35 \\ 2335 & - \\ \mathrm{I} 4 / 5 & \text { 10 } \\ - & -\end{array}$

Females

$\begin{array}{llr}+\mathrm{I} 3 / 5 & +\mathrm{I} & - \\ +\mathrm{I} 2 / 5 & +\mathrm{I} & - \\ +4 & +34 / 5 & 7 \\ +\mathrm{I} 4 / 5 & +\mathrm{I} & 7 \\ +4 & +32 / 5 & \mathrm{rO} \\ +33 / 5 & +31 / 5 & \mathrm{I} 4 \\ +4 & +31 / 5 & 56 \\ +2 & +\mathrm{I} 4 / 5 & \mathrm{I} 4 \\ +\mathrm{I}^{2} / 5 & +4 / 5 & 3 \\ +24 / 5 & +23 / 5 & - \\ +32 / 5 & +24 / 5 & 7 \\ +4 & +34 / 5 & \mathrm{I} 6 \\ +2 & +\mathrm{I} 4 / 5 & -\end{array}$




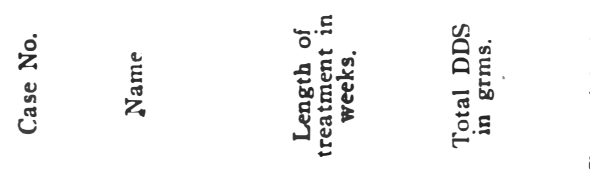

Budhu

Dhanonjoy … 50 $50 \frac{1}{2} \quad 20.2$

Umesh $\quad 44 \frac{1}{2} \quad 17.8$

Umesh

Premsaran ...

Sashee

Domon

Chuttu

Satrughna

Sripeti

Doyasagar ...

Budhan

Robi

Ganesh

Dinobondhu.

IOf Nodu

\section{9}

$5 \mathrm{I} \frac{1}{\mathrm{~s}} \quad 20.6$

20.4

20.4
I6.4

16.4
19.4

I6.0

20.4

20.4

I 8.8

20.2
19.6

I 9.6

20.4

I9. 2
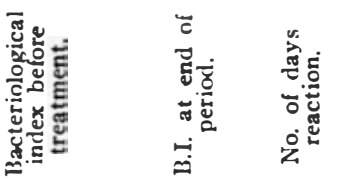

\section{$+3 / 5$}

+4
$+11 / 5$

$+11 / 5$
$+11 / 5$

$+4$

+1
+3

$+22 / 5$

$+4$

+4
+4
+4

+4
$+33 / 5$
$+24 / 5$

$+24 / 5$

$+4$

+4
+4

$+3 / 5 \quad 7$

$\begin{array}{ll}+4 & 42 \\ + & \\ +115 & 10 \\ + & \end{array}$

$+11 / 5$

+4
+1

+1
+3
+

+3
$+22 / 5$

$+2 \%$
+4

+4
+4
+4

+4
$+33 / 5$
+245

$34 / 5$
+

+4
+4

$+4$

\begin{tabular}{|c|c|c|c|c|c|c|}
\hline Rashu & $\cdots$ & 48 & 19.2 & $+13 / 5$ & +3 & 2 I \\
\hline Dulal & ... & 50 & 20.4 & $+\mathrm{I}$ & $+11 / 5$ & - \\
\hline Usman & ... & $48 \frac{1}{2}$ & I9.4 & $+31 / 5$ & $+33 / 5$ & $I_{4}$ \\
\hline Phakir & $\cdots$ & $48 \frac{1}{2}$ & I9. 4 & +2 & $+22 / 5$ & $2 I$ \\
\hline Ismael & $\cdots$ & $47 \frac{1}{2}$ & I9.0 & $+31 / 5$ & $+34 / 5$ & 10 \\
\hline Sashee & $\cdots$ & $5 \mathrm{I} \frac{1}{2}$ & 20.6 & $+21 / 5$ & $+31 / 5$ & - \\
\hline Motilal & $\cdots$ & $5 I$ & 20.4 & $+31 / 5$ & $32 / 5$ & - \\
\hline Joseph & $\cdots$ & $5 I$ & 20.4 & $+14 / 5$ & $+22 / 5$ & - \\
\hline & $\cdots$ & 51 & 20.4 & $+21 / 5$ & $+34 / 5$ & 一 \\
\hline
\end{tabular}

WORSE
そ்

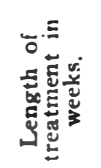

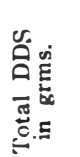

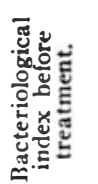

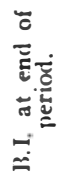

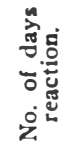

$\begin{array}{rllll}3 & \text { Rotni } & \ldots & 48 & 19.2 \\ 5 & \text { Sashee } & \ldots & 50 & 20.4 \\ 4 \text { I } & \text { Dashi } & \ldots & 5 \text { I } \frac{1}{2} & 20.6 \\ 44 & \text { Chepi } & \ldots & 47 \frac{1}{2} & 19.4\end{array}$

+4
+4
+4
+4

$\begin{array}{ll}+4 & 4 \\ +4 & - \\ +4 & - \\ +4 & \text { r }\end{array}$

$\begin{array}{lllllrrr}\text { I I } & \text { Kodom } & \ldots & 4 \mathrm{I} & \text { I } 6.4 & +2 \mathrm{I} / 5 & +3 & 52 \\ \text { I5 } & \text { Bhadu } & \ldots & 5 \mathrm{I} \frac{1}{2} & 20.6 & +32 / 5 & +33 / 5 & - \\ \text { I6 } & \text { Subodhani } & \ldots & 49 \frac{1}{2} & 19.8 & \text { neg. } & \text { 2 bac. } & - \\ \text { 2 I } & \text { Dongi } & \ldots & 48 \frac{1}{2} & \text { I9.4 } & +24 / 5 & +32 / 5 & \text { I4 } \\ 33 & \text { Rosoana } & \ldots & 45 & \text { I8.0 } & + \text { II } 15 & +31 / 5 & 45 \\ 40 & \text { Ulia } & \ldots & 49 \frac{1}{2} & 19.8 & +31 / 5 & +4 & 3 \\ 45 & \text { Beji } & \ldots & 5 \mathrm{I} & 20.4 & +4 / 5 & + \text { I } & -\end{array}$


and sex. Cochrane used .5 grm. to 2.5 grm. per week in most of his cases. Molesworth used .2 grm. to I grm. per week.

\section{LEPRA RIACTION.}

Eighty-nine cases, or $63.5 \%$, suffered from reaction. Cases F. 53, 25, II, I5, 33 suffered most from $77,52,52,52,45$ days respectively; of these 3 improved slightly and 2 became worse. Cases M. 56, 4I, 90, 47, I5 suffered from 66, 63, 56, 4542 days respectively. Three improved slightly, 3 remained stationary and none became worse.

In the reactionary stages while being treated with the parent sulphone, the nodules have been observed as diffuse swellings. Discrete nodules were fewer in acute reaction. In 4 cases the swellings softened and, on incision, thick pus came out. On staining, the specimen contained M. leprae and pus cells only. In one case, most of the eruptions ulcerated.

\section{SUMMARY.}

One hundred and forty cases have been treated with $20 \%$ DDS suspension for I year. Groundnut oil, hydnocarpus oil and refined deodorised cocoanut oil have been used as suspending agents. Bacteriological improvements are shown in Tables $I$ and 2 in short and in detail, sex by sex: Lepra reaction has been discussed. Depot formation has been observed.

\section{CONClusions.}

One hundred and four, or $74.1 \%$ of cases, have improved with a dose of .4 grm. per week for one year. There was very little difficulty and treatment could be continued easily without any supplementary treatment, to almost all. Except in 4 cases out of 89 , or $63 \%$, reaction cases needed very little special care for the continuation of treatment. DDS can be suspended easily in any vehicle. Refined hydnocarpus oil costs only two-thirds of the purified cocoanut oil, and depots may be seen with any of the vehicles used for the suspension of DDS, but more particularly when arachis oil is used.

\section{REFERENCES.}

I. Leprosy Review, Vol. XX, I949, p. 4. Cochrane, Ramanujam and Paul.

2. International Journal of Leprosy, Vol. 17, 1949, p. I97. Molesworth and Narayanswami. 